\title{
Conforto térmico humano em escritórios com sistema central de condicionamento artificial em clima subtropical úmido: estudos de campo vs. abordagem analítica
}

\author{
Human thermal comfort in a HVAC office building located \\ in a humid subtropical climate: field studies vs. \\ analytical approach
}

Ricardo Forgiarini Rupp Universidade Federal de Santa Catarina

Florianópolis - SC - Brasil

Renata De Vecchi Universidade Federal de Santa Catarina Florianópolis - SC - Brasil

Bernardo Farias Asmus Universidade Federal de Santa Catarina

Florianópolis - SC - Brasil

Christhina Maria Cândido The University of Sydney Sydney - Austrália

Enedir Ghisi Universidade Federal de Santa Catarina Florianópolis - SC - Brasil

Recebido em 26/03/16 Aceito em 29/09/16

\section{Ricardo Forgiarini Rupp \\ Renata De Vecchi \\ Bernardo Farias Asmus \\ Christhina Candido \\ Enedir Ghisi}

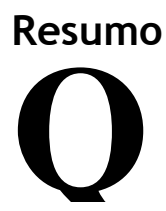

uando se trata de conforto térmico em edificações condicionadas artificialmente, o modelo predicted mean vote/predicted percentage of dissatisfied (PMV/PPD) de Fanger, publicado em 1970, é o mais utilizado para prever e avaliar as condições térmicas internas. Este artigo apresenta dados de conforto térmico levantados em uma edificação de escritórios com sistema central de condicionamento de ar, localizada em Florianópolis, uma cidade de clima subtropical úmido. O objetivo da pesquisa é analisar e comparar os resultados de sensação térmica obtidos em estudos de campo (284 participantes) com os valores calculados de PMV/PPD provenientes do método analítico adotado pela ASHRAE 55 (2013). Questionários eletrônicos foram aplicados simultaneamente às medições das variáveis ambientais (temperatura do ar, umidade relativa, temperatura radiante média e velocidade do ar) durante 2014. Observou-se que, embora $91 \%$ dos ocupantes tenham avaliado o ambiente como confortável termicamente, o PPD médio apontou $16 \%$ de insatisfeitos termicamente. Constatou-se certa inadequação do modelo ao clima em questão, principalmente quando se considera o restrito intervalo de PMV entre \pm 0,50 delimitado como confortável pela ASHRAE 55 (2013).

Palavras-chaves: Conforto térmico. Escritórios. PMV. PPD. Estudo de campo.

\section{Abstract}

When it comes to thermal comfort in fully air conditioned buildings, Fanger's predictive mean vote/predicted percentage of dissatisfied (PMV/PPD) published in 1970 is the most widely used model to predict and analyse environments. This paper presents thermal comfort data from an office building that operates with a central air-conditioning system (HVAC) located in Florianópolis, a city with humid subtropical climate on the southern coast of Brazil. This paper presents and compares thermal sensation results obtained from field studies (284 participants) against calculated PMV/PPD values based on the ASHRAE 55 (2013) analytical method. Electronic questionnaires were administered simultaneously to instrumental measurements (air temperature, radiant air temperature, air speed and humidity) during 2014. The results showed that, although $91 \%$ of the occupants evaluated the environment as thermally comfortable, the mean PPD scored $16 \%$ of dissatisfied people. Moreover, results suggested a certain inadequacy of this model to the climate in question, especially when the PMV ranges between \pm 0.50 , the interval determined as comfortable by ASHRAE 55 (2013).

Keywords: Thermal comfort. Offices. PMV. PPD. Field study.

RUPP, R. F.; DE VECCHI, R.; ASMUS, B. F.; CANDIDO, C. M.; GHISI, E. Conforto térmico humano em escritórios com sistema central de condicionamento artificial em clima subtropical úmido: estudos de campo vs. abordagem analítica. Ambiente Construído, Porto Alegre, v. 17, n. 1, p. 111-123, jan./mar. 2017. ISSN 1678-8621 Associação Nacional de Tecnologia do Ambiente Construído. http://dx.doi.org/10.1590/s1678-86212017000100127 


\section{Introdução}

Na década de 1970, P. O. Fanger apresentou um método analítico que prevê, para diferentes tipos de atividade metabólica e vestimenta de ocupantes, as combinações de variáveis térmicas ambientais internas que resultam na maior porcentagem possível de pessoas em conforto térmico dentro de um mesmo espaço (FANGER, 1970). Esse modelo, baseado em estudos executados em câmaras climáticas durante o inverno na América do Norte e validado por meio de outros estudos em câmara climática realizados durante o outono na Dinamarca, avalia a sensação térmica a partir de dois índices complementares: o predicted mean vote (PMV), ou voto médio estimado, que descreve a sensação térmica média de um grupo de pessoas ocupando o mesmo ambiente, e o predicted percentage of dissatisfied (PPD), ou porcentagem predita de insatisfeitos, que determina a quantidade de pessoas insatisfeitas nesse mesmo ambiente com base no PMV. Atualmente, tal modelo representa um dos principais métodos de avaliação de conforto térmico nas normas internacionais da área, como a ISO 7730 (INTERNATIONAL..., 2005) e a ASHRAE 55 (AMERICAN..., 2013), e é essencialmente indicado para avaliações gerais de conforto térmico em que a velocidade do ar não ultrapassa $0,20 \mathrm{~m} / \mathrm{s}$ (o que comumente é observado em espaços condicionados artificialmente).

Com o passar do tempo, e a concretização de um número cada vez maior de estudos de campo, um novo método de avaliação chamado modelo adaptativo (DE DEAR; BRAGER, 1998; HUMPHREYS; RIJAL; NICOL, 2013; NICOL; HUMPHREYS, 2010, 2002) evidenciou algumas limitações do modelo de Fanger, com viés mais acentuado em ambientes ventilados naturalmente (HUMPHREYS; NICOL, 2002; VAN HOOF, 2008; DEUBLE; DE DEAR, 2012). De acordo com outros estudos, o modelo de Fanger pode também apresentar inadequação em espaços condicionados artificialmente de climas subtropicais durante os períodos mais quentes, como em Taiwan (HWANG et al., 2009), Hong Kong (FONG; CHOW; LI, 2010) e Índia (MAITI, 2013, 2014). Na Malásia, o super-resfriamento foi indicado como o principal problema observado nas comparações entre os votos reais e os votos estimados (DAGHIGH; SOPIAN, 2009). No norte da Itália (RICCIARDI; BURATTI, 2012), na China (CAO et al., 2011) e na Bélgica (HENS, 2009), estudos de campo encontraram fraca correlação entre o voto médio de sensação térmica dos usuários (actual mean vote (AMV), ou voto médio real) e o PMV, enquanto outros estudos demonstraram discrepância entre os valores de
PMV e AMV. Porém, na Alemanha, um estudo mostrou que durante o inverno, estação climática na qual o PMV foi desenvolvido com pessoas da América do Norte, e em 25 edifícios de escritórios em 11 cidades diferentes, o PMV apresentou valores muito próximos às respostas subjetivas dos ocupantes (KUCHEN; FISCH, 2009). Outros estudos comparativos entre o PMV e o AMV podem ser consultados no artigo de revisão da literatura de Rupp, Vásquez e Lamberts (2015).

No Brasil, poucos resultados provenientes de estudos de campo foram publicados com foco na adequabilidade do modelo PMV/PPD. Nesses, as maiores discrepâncias observadas foram com relação à porcentagem de pessoas insatisfeitas: o PPD superestima a quantidade de insatisfeitos em um ambiente (LAMBERTS et al., 2013). Segundo os autores, as limitações do modelo no contexto brasileiro existem especialmente em climas quentes e úmidos, nos quais o modelo tende a superestimar o PPD entre $15 \%$ e $20 \%$, e inclusive o PMV, tanto para a escala de calor, quanto de frio. Andreasi, Lamberts e Cândido (2010) encontraram certa proximidade entre os votos de sensação térmica dos ocupantes de uma edificação condicionada artificialmente e o PMV calculado em uma região de clima quente e úmido (Campo Grande, MS). No entanto, observou-se que no período mais quente do estudo (novembro), o PPD superestimou a porcentagem real de pessoas insatisfeitas (actual percentage of dissatisfied (APD)) no ambiente em $13 \%$.

Com base nessa discussão, é possível concluir que quando se trata de conforto térmico em espaços internos condicionados artificialmente, pode haver discrepâncias entre o que é predito e o que é real. Tais discrepâncias ocorrem com frequência nas edificações de climas mais quentes ou durante os períodos mais quentes em edificações de climas amenos, independentemente do modo de climatização em operação no ambiente (HWANG et al., 2009; HENS, 2009; FONG; CHOW; LI, 2010; CAO et al., 2011; RICCIARDI; BURATTI, 2012; MAITI, 2013, 2014). Mesmo que a inadequação do modelo de Fanger (1970) já tenha sido discutida anteriormente no Brasil, o principal objetivo deste artigo é apresentar uma análise dos dados de conforto térmico levantados em Florianópolis, uma ilha com clima subtropical úmido. Os resultados focaram ainda na adequação do método proveniente do PMV e do PPD da ASHRAE 55 (AMERICAN..., 2013) durante as estações de outono, inverno e primavera. As análises se basearam em 1.235 questionários coletados em uma edificação de escritórios

112 Rupp, R. F.; De Vecchi, R.; Asmus, B. F.; Candido, C. M.; Ghisi, E. 
localizada em Florianópolis, que utiliza um sistema de ar condicionado central durante todo o ano.

\section{Método}

Este artigo baseia-se em uma análise comparativa entre os valores estimados de conforto térmico, calculados a partir do modelo de Fanger (PMV/PPD) e aqueles reportados pelos usuários de uma edificação de escritórios (AMV/APD). Os dados analisados foram coletados em campo durante o ano de 2014, nos períodos de outono, inverno e primavera, totalizando 25 medições em ambientes diferentes (Tabela 1). Os procedimentos adotados incluíram medições das variáveis ambientais (temperatura do ar, de globo, umidade relativa e velocidade do ar) realizadas simultaneamente ao preenchimento de questionários eletrônicos (sensação, preferência e aceitabilidade térmica e da velocidade do ar) aplicados aos usuários.

A edificação onde os estudos de campo foram realizados está localizada em Florianópolis, capital de Santa Catarina (latitude 27,59 $\mathrm{S}$, longitude $48,54^{\circ} \mathrm{O}$ ), onde o clima se caracteriza por verões quentes e invernos razoavelmente frios (clima subtropical úmido). $\mathrm{Na}$ edificação trabalham diariamente cerca de 1.200 pessoas. A edificação possui cinco pavimentos, sendo dois em subsolo. $\mathrm{O}$ primeiro e o segundo pavimentos foram investigados neste trabalho, obtendo-se 1.235 votos de conforto térmico (n) provenientes de 284 funcionários que participaram voluntariamente dos estudos (alguns usuários não responderam as cinco rodadas de perguntas por motivos diversos reunião de trabalho fora do espaço estudado, consulta médica, saída para trabalho em campo, dentre outros). A edificação, que em 2007 passou por uma reforma interna e recebeu selo Procel Edifica nível A, funciona predominantemente com um sistema central de condicionamento artificial, operando de segunda a sexta-feira entre $7 \mathrm{~h}$ e $19 \mathrm{~h}$. Os espaços internos possuem planta livre, e são delimitados por divisórias comuns de madeira (de piso a teto), que eventualmente são remoduladas. Nos ambientes onde os estudos foram realizados, os ocupantes não possuem nenhum tipo de controle sobre as condições térmicas, e a temperatura do ar é mantida em $24^{\circ} \mathrm{C}$, com variação aceitável de $\pm 2^{\circ} \mathrm{C}$. A umidade interna não é controlada, e a saída de ar ocorre através de frestas modulares $(1 \times 1 \mathrm{~m})$ localizadas no teto. Foi acordado com a empresa que as informações coletadas no edifício são sigilosas. Portanto, não serão mostradas plantas nem localização da edificação estudada.

Tabela 1 - Identificação dos 25 estudos de campo: data, período e estação climática

\begin{tabular}{l|c|c|c|c|c}
\hline Estudo de campo & 1 & 2 & 3 & 4 & 5 \\
\hline Data & $31 / 03 / 2014$ & $03 / 04 / 2014$ & $03 / 04 / 2014$ & $09 / 04 / 2014$ & $09 / 04 / 2014$ \\
\hline Período & Manhã & Manhã & Tarde & Manhã & Tarde \\
\hline Estação climática & Outono & Outono & Outono & Outono & Outono \\
\hline Estudo de campo & 6 & 7 & 8 & 9 & 10 \\
\hline Data & $10 / 04 / 2014$ & $11 / 04 / 2014$ & $16 / 04 / 2014$ & $23 / 04 / 2014$ & $23 / 04 / 2014$ \\
\hline Período & Tarde & Manhã & Manhã & Manhã & Tarde \\
\hline Estação climática & Outono & Outono & Outono & Outono & Outono \\
\hline Estudo de campo & 11 & 12 & 13 & 14 & 15 \\
\hline Data & $05 / 05 / 2014$ & $07 / 05 / 2014$ & $07 / 05 / 2014$ & $09 / 05 / 2014$ & $16 / 07 / 2014$ \\
\hline Período & Tarde & Manhã & Tarde & Manhã & Manhã \\
\hline Estação climática & Outono & Outono & Outono & Outono & Inverno \\
\hline Estudo de campo & 16 & 17 & 18 & 19 & 20 \\
\hline Data & $08 / 08 / 2014$ & $08 / 08 / 2014$ & $08 / 08 / 2014$ & $08 / 08 / 2014$ & $20 / 08 / 2014$ \\
\hline Período & Manhã & Manhã & Tarde & Tarde & Manhã \\
\hline Estação climática & Inverno & Inverno & Inverno & Inverno & Inverno \\
\hline Estudo de campo & 21 & 22 & 23 & 24 & 25 \\
\hline Data & $20 / 08 / 2014$ & $27 / 08 / 2014$ & $27 / 08 / 2014$ & $09 / 10 / 2014$ & $09 / 10 / 2014$ \\
\hline Período & Manhã & Manhã & Manhã & Manhã & Tarde \\
\hline Estação climática & Inverno & Inverno & Inverno & Primavera & Primavera \\
\hline
\end{tabular}




\section{Sensações dos usuários}

\section{Instrumento de coleta de dados}

O questionário eletrônico utilizado neste estudo foi desenvolvido pelo Laboratório de Eficiência Energética em Edificações (LabEEE/UFSC), e inclui perguntas sobre os dados antropométricos e demais características individuais dos usuários (vestimenta e atividade metabólica), sensação, preferência e aceitabilidade térmica, conforto térmico e movimento do ar (preferência e aceitabilidade). As seguintes escalas foram utilizadas no questionário:

Qual é a sua sensação térmica neste momento? 3 muito frio; -2 frio; - 1 levemente com frio; 0 neutro; +1 levemente com calor; +2 calor; +3 com muito calor.

Você preferiria estar: +1 mais resfriado; 0 assim mesmo; -1 mais aquecido.

Para você este ambiente térmico é: 0 aceitável; 1 inaceitável.

Neste momento, você está: O confortável; 1 desconfortável.

Com relação à velocidade do ar neste momento, está: 0 aceitável ("pouca velocidade do ar", "suficiente velocidade do ar" e "muita velocidade do ar"); 1 inaceitável ("pouca velocidade do ar" $e$ "muita velocidade do ar").

Considerando a sua resposta anterior, qual sua preferência com relação ao movimento do ar neste momento? 2 menos velocidade do ar; 1 não mudar; 0 mais velocidade do ar.

Na questão sobre conforto térmico, os usuários podiam escolher entre as opções "confortável" e "desconfortável"; se a opção escolhida fosse "desconfortável”, os usuários podiam indicar o motivo do desconforto (por exemplo: "meus pés estão frios"). Sobre a aceitabilidade da velocidade do ar, além dos usuários responderem se ela era "aceitável” ou "inaceitável", solicitou-se ainda que eles apontassem o motivo pelo qual a classificação escolhida era aceitável ou não: se aceitável, por "pouca velocidade do ar", "suficiente velocidade do ar" ou "muita velocidade do ar"; se inaceitável, por "pouca velocidade do ar" ou "muita velocidade do ar" (CÂNDIDO et al., 2010). Anexo ao e-mail com o questionário e convite para participar da pesquisa, os funcionários recebiam também uma carta explicativa, com informações adicionais a respeito dos procedimentos de medição e de como utilizar o questionário.

\section{Ocupantes da edificação}

Os funcionários da edificação foram recrutados pelos pesquisadores e pelo chefe de setor para participar voluntariamente do estudo de campo. As características antropométricas dos voluntários podem ser observadas na Tabela 2. Dentre os 284 usuários que responderam ao questionário, $63 \%$ são do gênero masculino e $37 \%$ do gênero feminino. Os valores de resistência térmica da vestimenta e taxa metabólica foram estimados de acordo com a ASHARE 55 (AMERICAN..., 2013). Aos valores de resistência térmica da vestimenta dos usuários foi incluído um adicional de $+0,04$ clo relativos a roupa íntima e outro adicional de $+0,10$ clo referente ao isolamento das cadeiras de escritório. $\mathrm{Na}$ Tabela 2 também podem ser visualizados os dados de isolamento de vestimenta e de metabolismo da população estudada.

\section{Instrumentos de medição}

A temperatura do ar $\left({ }^{\circ} \mathrm{C}\right)$, umidade relativa $(\%)$, temperatura de globo $\left({ }^{\circ} \mathrm{C}\right)$ e velocidade do ar $(\mathrm{m} / \mathrm{s})$ foram medidos a partir de duas estações microclimáticas SENSU desenvolvidas pelo Laboratório de Meios Porosos e Propriedades Termofísicas da Universidade Federal de Santa Catarina (Figura 1). Além das estações microclimáticas, também foram usados dois termoanemômetros portáteis para medições pontuais de temperatura e velocidade do ar, da marca AirFlow, modelo TA35 (Figura 2). Todas as medições foram realizadas conforme as especificações para medições de conforto térmico do apêndice $\mathrm{K}$ da ASHRAE 55 (AMERICAN..., 2013).

Tabela 2 - Perfil antropométrico, isolamento da vestimenta e taxa metabólica dos participantes nos estudos de campo

\begin{tabular}{l|c|c|c|c|c}
\hline Parâmetro & Peso (kg) & Idade (anos) & Altura (m) & Vestimenta (clo) & Metabolismo (met) \\
\hline Média & 75,0 & 38,8 & 1,72 & 0,68 & 1,04 \\
Desvio padrão & 14,5 & 11,2 & 0,09 & 0,16 & 0,09 \\
Máxima & 108,0 & 65,0 & 1,97 & 1,40 & 1,40 \\
Mínima & 42,0 & 17,0 & 1,50 & 0,42 & 1,00 \\
\hline
\end{tabular}

114 Rupp, R. F.; De Vecchi, R.; Asmus, B. F.; Candido, C. M.; Ghisi, E. 
Figura 1 - Estação microclimática SENSU

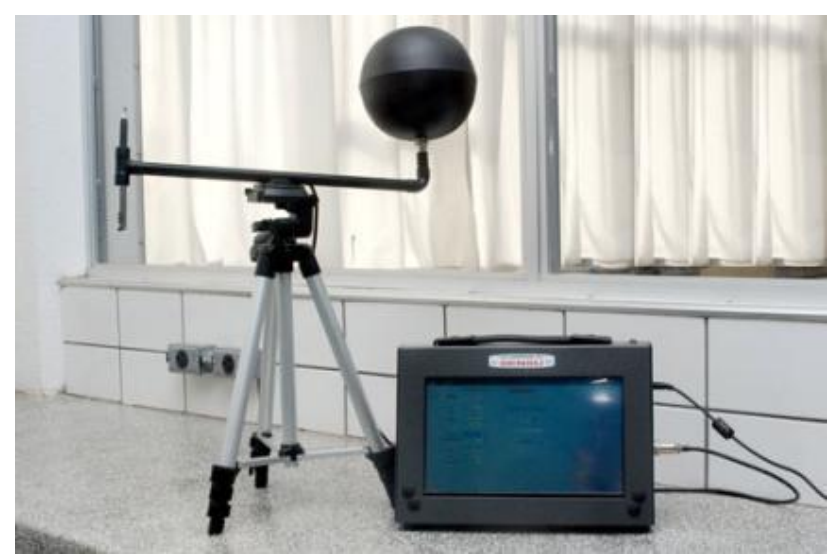

Figura 2 - Termo anemômetro portátil AirFlow

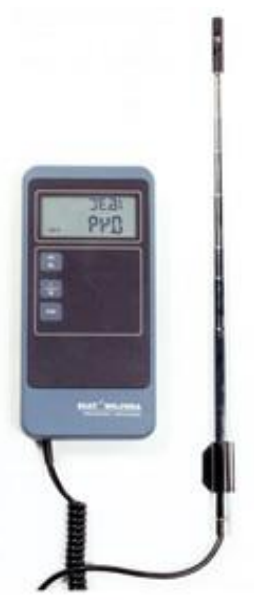

\section{Procedimentos de medição e análise dos dados}

Durante os estudos de campo, os ocupantes foram solicitados a avaliar sua percepção térmica nas estações de trabalho respondendo ao questionário uma vez a cada 20 minutos, o que totalizou cinco rodadas de perguntas em um período de aproximadamente 1 hora e 40 minutos de medição. Previamente a esse intervalo, foram utilizados cerca de 30 minutos para a estabilização dos sensores e do nível de metabolismo dos ocupantes. Ao mesmo tempo em que os ocupantes respondiam aos questionários, e as medições das variáveis ambientais eram registradas pelas estações microclimáticas, observações acerca do ambiente e comportamento dos usuários foram realizadas.

As medições das variáveis ambientais foram efetivadas de maneira setorizada, com o confortímetro localizado continuamente em um ponto central da sala onde os ocupantes estavam respondendo aos questionários. Em todas as medições, os procedimentos para a obtenção dos dados foram os mesmos, os quais podem ser observados mais detalhadamente na Figura 3.

A temperatura operativa $\left(\mathrm{T}_{\mathrm{o}}\right)$ foi calculada por meio da Equação 1, de acordo com a ASHRAE 55 (AMERICAN..., 2013), enquanto o PMV e o PPD foram calculados utilizando-se a calculadora online do Center for the Built Environment (CBE) desenvolvida por Hoyt et al. (2013).

$\mathrm{T}_{\mathrm{o}}=\mathrm{A} \cdot \mathrm{t}_{\mathrm{ar}}+(1-\mathrm{A}) \cdot \mathrm{t}_{\mathrm{rm}}$

Eq. 1

Onde:

$\mathrm{A}=0,5$ para $\mathrm{V}_{\mathrm{ar}}$ menor que $0,2 \mathrm{~m} / \mathrm{s}$;

$\mathrm{A}=0,6$ para $\mathrm{V}_{\mathrm{ar}}$ de 0,2 a $0,6 \mathrm{~m} / \mathrm{s}$;

$\mathrm{A}=0,7$ para $\mathrm{V}_{\mathrm{ar}}$ de 0,6 a $1,0 \mathrm{~m} / \mathrm{s}$;

$\mathrm{t}_{\mathrm{ar}}$ é a temperatura do ar $\left[{ }^{\circ} \mathrm{C}\right]$; e

$\mathrm{t}_{\mathrm{rm}}$ é a temperatura média radiante $\left[{ }^{\circ} \mathrm{C}\right]$. 
Figura 3 - Protocolo de medição adotado durante a realização dos estudos de campo

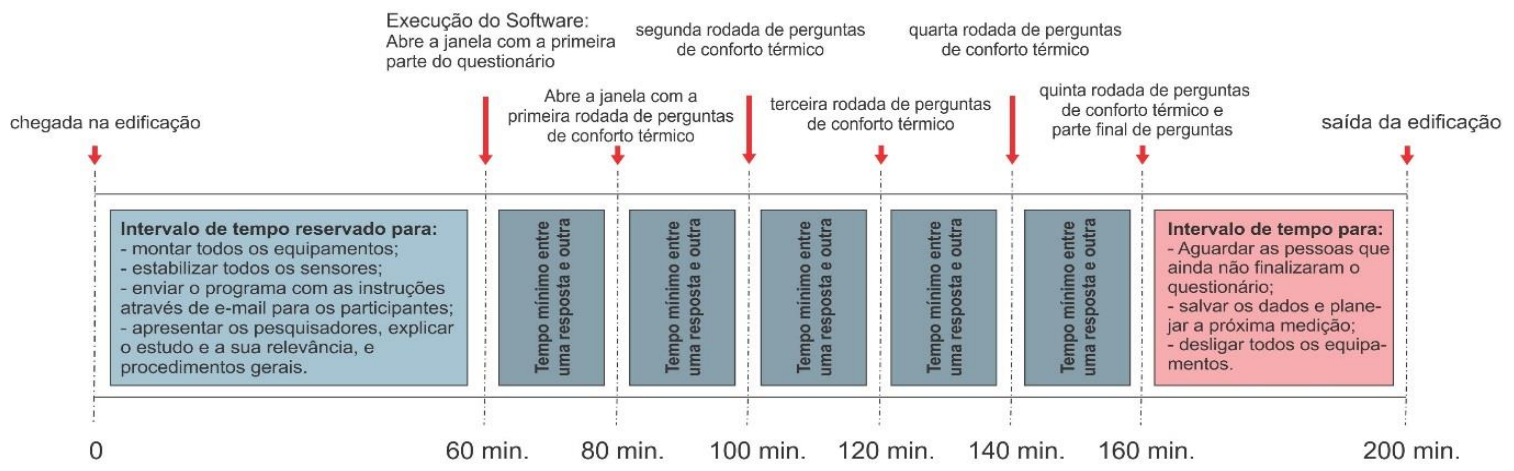

Duração de cada medição / Período do dia

\section{Resultados}

\section{Variáveis ambientais internas}

Um resumo das condições térmicas internas (temperatura operativa, umidade relativa e velocidade do ar) durante os estudos de campo pode ser observado na Tabela 3 . A temperatura operativa variou pouco durante os estudos de campo, pois o sistema central de condicionamento de ar manteve condições praticamente constantes de temperatura (média de $22,9^{\circ} \mathrm{C}$ ). De modo geral, a velocidade do ar apresentou valores baixos (média de $0,12 \mathrm{~m} / \mathrm{s}$ ), com pouca variabilidade no decorrer dos estudos de campo. O parâmetro que apresentou maior variação foi a umidade relativa do ar, que oscilou aproximadamente entre 23,0 e $78,0 \%$. Analisando as condições internas, pode-se afirmar que os dados não se encontram no intervalo da zona de conforto térmico estipulada pela ASHRAE 55 (AMERICAN..., 2013) - PMV médio localizado dentro do intervalo de $\pm 0,50-$ como se observa na Tabela 3. Embora o PMV calculado não se enquadre no intervalo estipulado pela norma, o voto médio real das pessoas (AMV) encontrado foi de $-0,23$.

\section{Conforto térmico}

A Figura 4 apresenta a porcentagem de votos de sensação térmica dos usuários da edificação em estudo, separadas por estação do ano e total. $\mathrm{Na}$ Figura 4 também podem ser visualizados os números de observações $(\mathrm{n})$ por período do ano. A maioria dos votos $(58,0 \%)$ se concentrou na categoria "neutro", enquanto $30,4 \%$ dos usuários disseram estar sentindo o ambiente térmico levemente frio a muito frio, e apenas $11,6 \%$ reportaram sensações térmicas de levemente com calor ou com calor. Considerando-se os três pontos centrais da escala sétima de sensação térmica $(-1,0$ e +1) como aqueles que proporcionam aceitabilidade térmica às pessoas (e não entre \pm 0,50, como estipula a ASHRAE 55 (AMERICAN..., 2013), pode-se afirmar que 94,9\% dos usuários estavam satisfeitos com o ambiente térmico (valor muito próximo aos votos reais de aceitabilidade térmica apresentados na Tabela 4). Os valores médios de AMV podem ser visualizados na Tabela 3 .

Devido à pequena variação das temperaturas operativas, não houve correlação entre estas e os votos de sensação térmica. A maioria das pessoas (71,7\% dos votos) preferiu manter as condições atuais do ambiente térmico (Figura 5). Porém, uma parcela significativa $(18,0 \%$ dos votos $)$ dos usuários preferiria que o ambiente estivesse mais aquecido, enquanto $10,3 \%$ prefeririam que o ambiente estivesse mais resfriado.

Ao cruzarem-se os votos de sensação térmica (AMV), que oscilaram entre -3 e +2 durante os estudos de campo, com os votos de preferência térmica (Figura 6), observa-se um número superior de votos para "não mudar" na escala de calor $(+1 \mathrm{e}$ +2 ), quando comparados com os votos de "não mudar" na escala de frio $(-1,-2$ e -3$)$. Provavelmente isso ocorre porque o ambiente tem pouca variação interna de temperatura, e também porque os usuários têm expressado, com maior frequência, sensações de levemente frio, com frio ou com muito frio (Figura 4). $\mathrm{Na}$ escala de sensações térmicas entre frio (-2) e muito frio (-3), a grande maioria dos usuários preferiria um ambiente mais aquecido, e no grupo de votos que demonstraram "levemente com frio" (-1), metade dos usuários gostaria de manter as atuais condições ambientais e a outra metade preferiria que o ambiente estivesse mais aquecido. Nos pontos $+1 \mathrm{e}$ +2 , observou-se que, respectivamente, $27,0 \%$ e $20,0 \%$ dos usuários gostariam de "não mudar" o ambiente; nesse caso, tal preferência pode estar relacionada ao desejo de experimentar temperaturas mais altas em um ambiente 
condicionado artificialmente que mantém temperatura interna constante de forma rígida, sem relacioná-la à temperatura externa.

De modo geral, as condições térmicas internas foram julgadas pelos usuários como confortáveis e aceitáveis, conforme pode ser visualizado na Tabela 4, que apresenta pouca diferença entre os votos de aceitabilidade térmica $(94,8 \%)$ e conforto térmico $(90,8 \%)$. Dentro da pequena amostra de ocupantes desconfortáveis termicamente $(9,2 \%$ dos votos), a grande maioria das reclamações é proveniente do desconforto por frio $(82,5 \%$ dos votos). Cerca de $90 \%$ das pessoas expressaram frio localizado nas extremidades do corpo (mãos e/ou pés), que poderia ser amenizado por meio do uso de pequenos sistemas de condicionamento personalizado, como os aquecedores portáteis para mãos ou pés (VESELY; ZEILER, 2014; ZHANG; ARENS; ZHAI, 2015).

Tabela 3 - Resumo das condições térmicas internas durante os estudos de campo

\begin{tabular}{l|c|c|c|c|c|c|c}
\hline Parâmetro & $\begin{array}{c}\text { Temperatura } \\
\text { operativa }\left({ }^{\circ} \mathbf{C}\right)\end{array}$ & $\begin{array}{c}\text { Umidade } \\
\text { relativa }(\boldsymbol{\%})\end{array}$ & $\begin{array}{c}\text { Velocidade do ar } \\
(\mathbf{m} / \mathbf{s})\end{array}$ & PMV & AMV & $\begin{array}{c}\text { PPD } \\
(\%)\end{array}$ & $\begin{array}{c}\text { APD } \\
(\%)\end{array}$ \\
\hline Média & 22,9 & 61,0 & 0,12 & $-0,61$ & $-0,23$ & 16 & 9 \\
Desvio padrão & 0,5 & 9,3 & 0,02 & 0,38 & 0,73 & 11 & 29 \\
Máxima & 24,5 & 78,0 & 0,30 & $+0,69$ & $+2,00$ & 75 & 100 \\
Mínima & 21,7 & 23,0 & 0,10 & $-1,94$ & $-3,00$ & 5 & 0 \\
\hline
\end{tabular}

Figura 4 - Votos de sensação térmica dos usuários

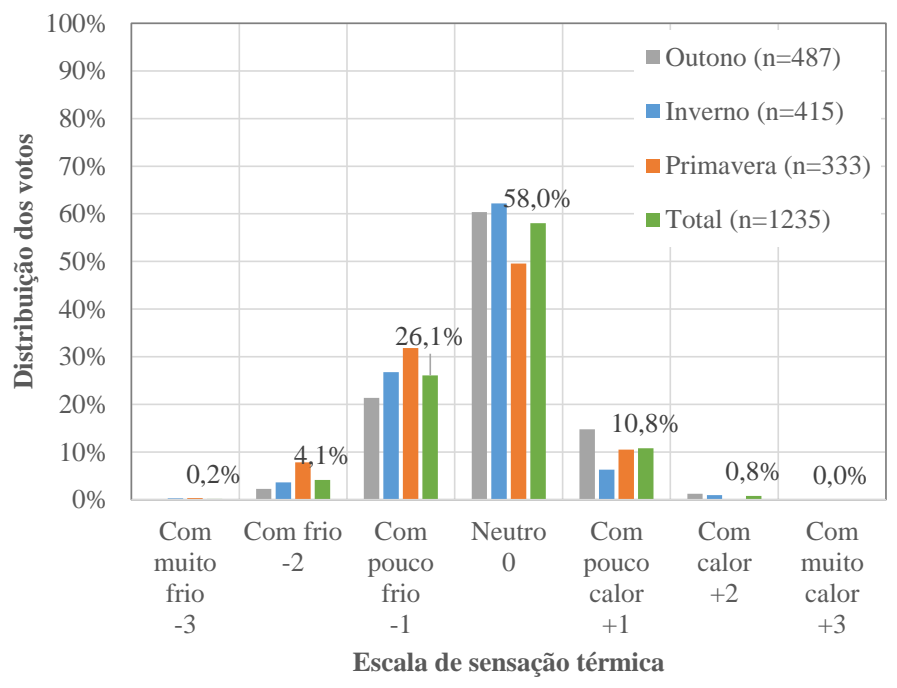

Figura 5 - Votos de preferência térmica dos usuários

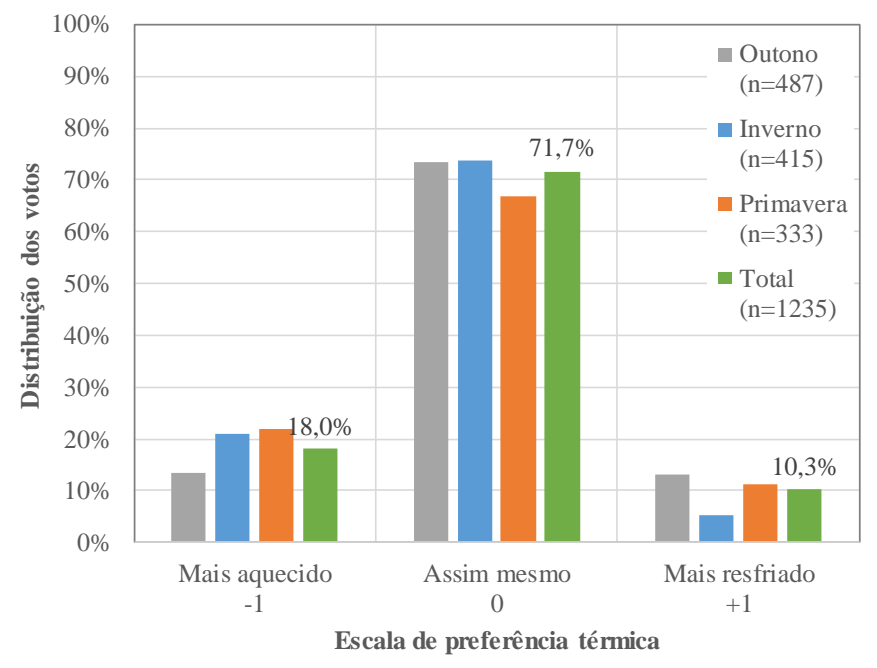

Conforto térmico humano em escritórios com sistema central de condicionamento artificial em clima subtropical úmido: estudos de campo vs. abordagem analítica 
Figura 6 - Sensação e preferência térmica dos usuários

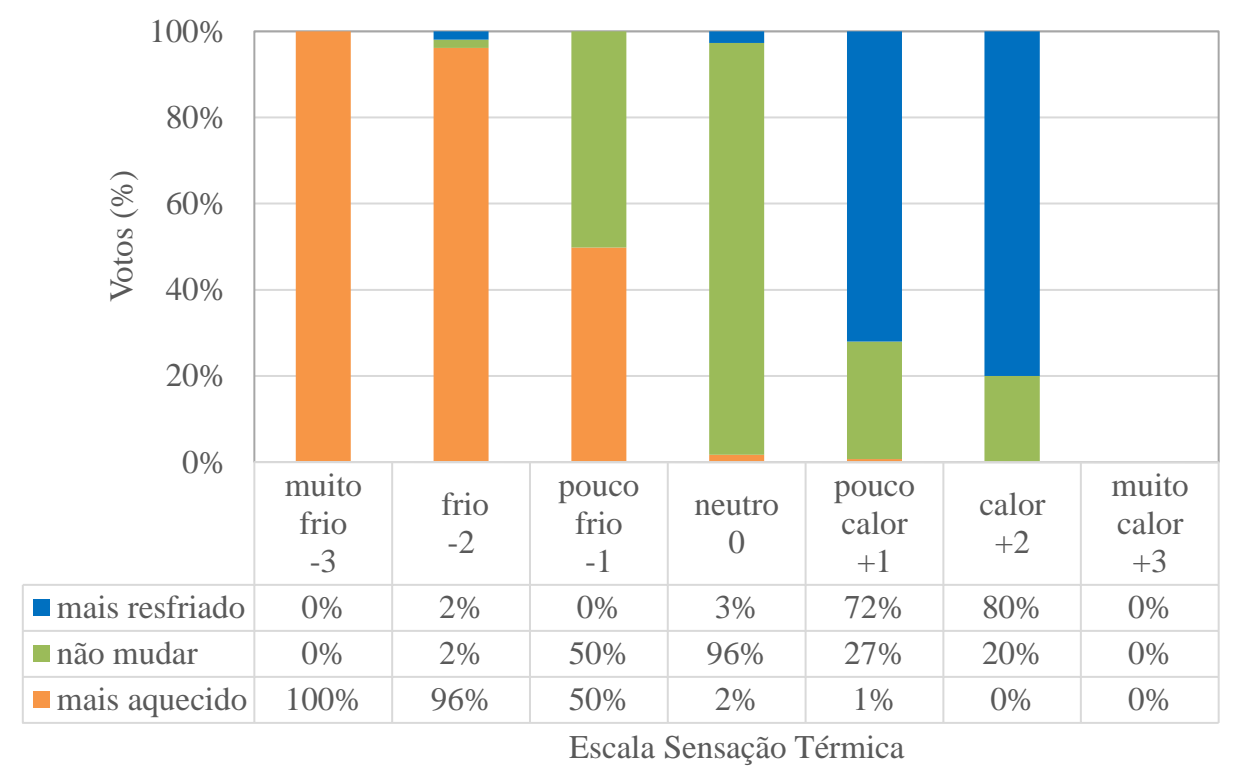

Tabela 4 - Aceitabilidade térmica e conforto térmico dos usuários

\begin{tabular}{l|c|c|c|c|c}
\hline Estação & $\mathbf{n}$ & Aceitável (\%) & Inaceitável (\%) & Confortável (\%) & Desconfortável (\%) \\
\hline Outono & 487 & 95,9 & 4,1 & 92,2 & 7,8 \\
Inverno & 415 & 93,3 & 6,7 & 89,4 & 10,6 \\
Primavera & 333 & 95,2 & 4,8 & 90,4 & 9,6 \\
Total & 1235 & 94,8 & 5,2 & 90,8 & 9,2 \\
\hline
\end{tabular}

Durante os estudos de campo, os usuários foram também questionados a respeito da aceitabilidade e preferência da velocidade do ar no seu espaço de trabalho. A velocidade média do ar durante os estudos de campo foi baixa $(0,12 \mathrm{~m} / \mathrm{s})$ e pouco variável (Tabela 3). A Figura 7 apresenta as respostas dos usuários com relação à velocidade do ar de acordo com o intervalo de temperatura operativa a que estavam sujeitos. A Figura 7a apresenta os votos de aceitabilidade da velocidade do ar $(n=1164)$, enquanto a Figura $7 b$ mostra os votos de inaceitabilidade da velocidade do ar $(n=71)$. Nos intervalos de temperatura operativa de 22 a $24^{\circ} \mathrm{C}$, observa-se que a maioria dos votos de inaceitabilidade ocorreu devido à baixa velocidade do ar. No entanto, dentre os votos de aceitabilidade da velocidade do ar apresentados na Figura 7a, em que a amostra é mais significativa, pode-se observar que a grande maioria dos usuários afirmou que a velocidade do ar era baixa, e por isso aceitável.

Com relação à preferência pela velocidade do ar (Figura 8), percebe-se que a grande maioria dos usuários votou por "não mudar" a velocidade do ar interna em todos os intervalos de temperatura. A porcentagem de pessoas que gostaria que a velocidade do ar fosse mais alta, ou fosse mais baixa, oscilou entre $11 \%$ e $13 \%$ e entre $2 \%$ e $13 \%$, respectivamente.

\section{Comparação entre os votos médios de sensação térmica preditos e reais}

A Figura 9 apresenta uma comparação entre os valores médios de AMV e PMV. A análise de variância (t-test), entre os resultados obtidos a partir dos dois métodos indicados, apontou diferença estatística $(\mathrm{p}<0,001)$ em todos os pontos analisados $\left(22^{\circ} \mathrm{C}, 23^{\circ} \mathrm{C}\right.$ e $\left.24^{\circ} \mathrm{C}\right)$. Observase que o PMV superestimou o voto médio calculado nos três intervalos de temperatura. A diferença entre o PMV médio calculado e o AMV real a $22^{\circ} \mathrm{C}$ foi de $-0,47$; a $23^{\circ} \mathrm{C}$, tal diferença foi de $-0,55$, e a $24^{\circ} \mathrm{C}$ a diferença foi de $-0,42$. É interessante notar que se o PMV fosse adotado neste estudo, conforme especifica a ASHRAE 55 (AMERICAN..., 2013), o ambiente seria classificado como "desconfortável" por apresentar PMV médio fora do intervalo entre $\pm 0,50$ (com exceção do intervalo de temperaturas próximas a $24^{\circ} \mathrm{C}$ ). No entanto, observou-se aproximadamente $90 \%$ de conforto térmico durante todo o estudo. 
Figura 7 - Aceitabilidade da velocidade ar em função da temperatura operativa

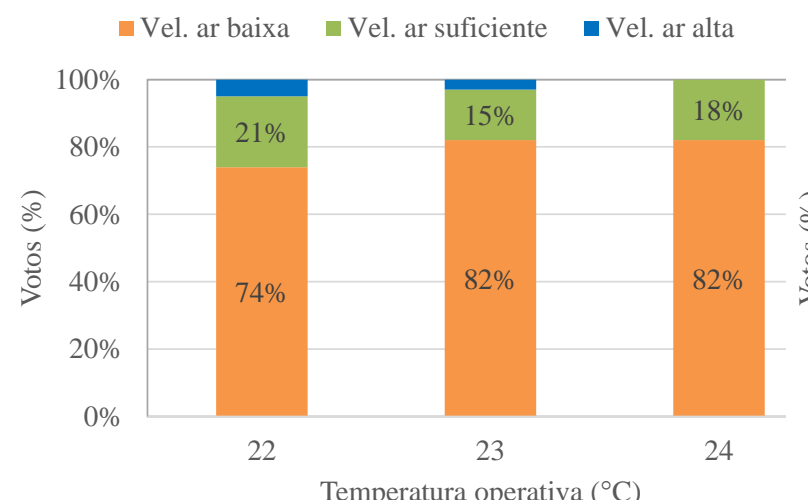

(a) Velocidade do ar aceitável
- Vel. ar muito alta $\quad$ Vel. ar muito baixa

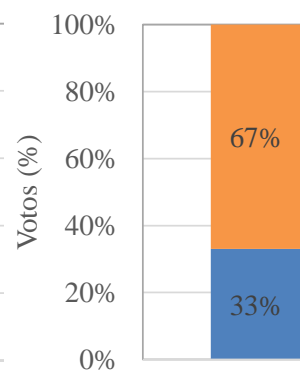

22

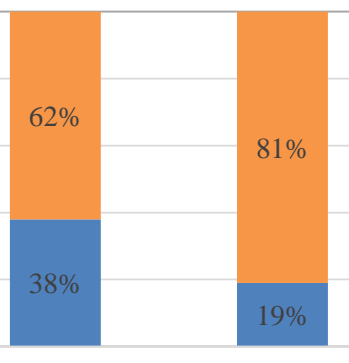

23
Temperatura operativa $\left({ }^{\circ} \mathrm{C}\right)$

(b) Velocidade do ar inaceitável

Figura 8 - Preferência dos usuários relacionada à velocidade do ar

- Mais velocidade $\quad$ Não mudar $\square$ Menos velocidade

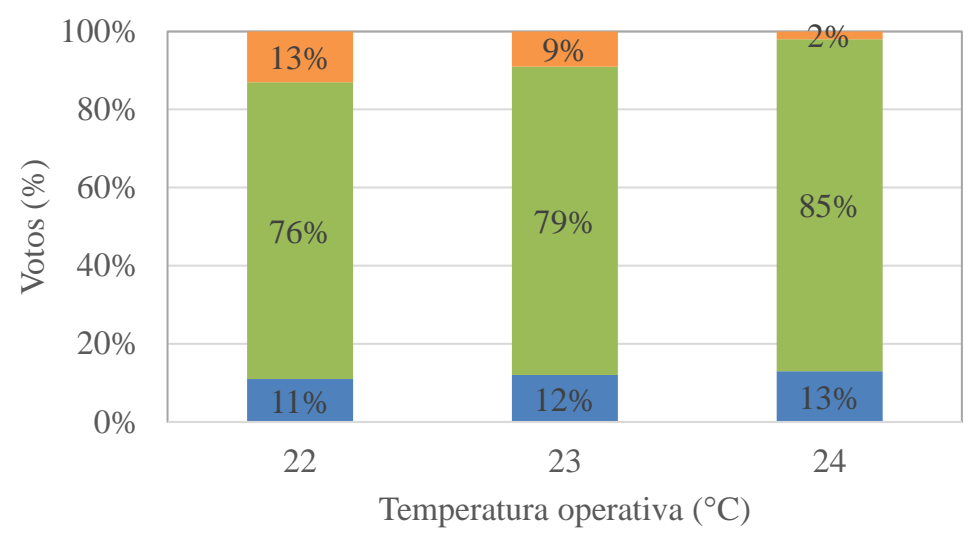

Figura 9 - Sensação térmica média entre o voto médio estimado (PMV) e o voto médio real (AMV)

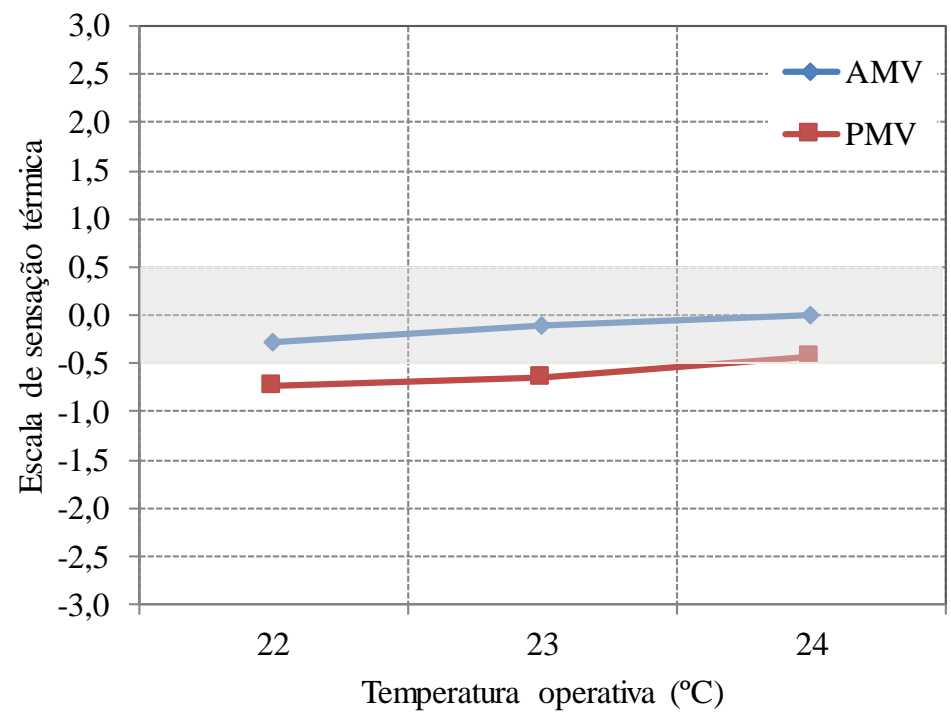


Os valores médios de PMV e AMV, quando comparados de acordo com os dias em que os dados foram levantados, variaram entre $-1,01$ (com frio) e $-0,22$ (neutro), e entre $-0,48$ (neutro) e $+0,18$ (neutro), respectivamente. A correlação entre o PMV e o AMV resultou em um coeficiente de determinação de 0,25 (Figura 10), enquanto todos os pontos se localizaram acima da bissetriz, que representa uma combinação perfeita entre o AMV e o PMV. A Figura 10 apresenta ainda a faixa de condições térmicas aceitáveis especificadas pela ASHRAE 55 (AMERICAN..., 2013), em que o PMV oscila entre $\pm 0,50$. Nota-se que somente $32 \%$ dos pontos estão em conformidade com a norma americana, ao mesmo tempo em que, na análise dos votos reais, $100 \%$ dos pontos cumprem com a norma se considerados os valores médios de AMV. A aceitabilidade térmica real das pessoas foi de $94,8 \%$.

O PMV superestimou a sensação de frio dos usuários, tendendo mais para o lado negativo da escala sétima, do que a real sensação das pessoas (nesse caso, a média das diferenças entre o PMV e o AMV foi de -0,44). Ainda que as médias apresentem os valores mais significativos para análise e comparação dos resultados, é interessante observar a variação de votos máxima e mínima entre o que é predito e o que é real. Enquanto os extremos na coluna dos votos reais de sensação térmica (AMV) chegaram a $+2,00$ na escala de calor, e -3,00 na escala de frio, o PMV previu sensação térmica máxima de $+0,69$ e mínima de -
1,94. Levando-se em consideração os resultados das Figuras 9 e 10, é notável a diferença entre o PMV e o AMV, e tais resultados podem ser ainda mais questionados se considerado o método analítico proveniente da ISO 7730 (INTERNATIONAL..., 2005) e ASHRAE 55 (AMERICAN..., 2013), que adota como confortável um estreito intervalo de sensação térmica média (PMV entre $\pm 0,50$ ).

A Figura 11 compara a porcentagem real de pessoas insatisfeitas (APD) com a porcentagem predita de insatisfeitos (PPD) em função do PMV para cada estudo de campo. $\mathrm{Na}$ maioria das situações o APD difere do PPD. A média das diferenças entre o PPD e o APD foi de 7\%; porém, houve diferenças de até $19 \%$. O PPD médio foi de $16 \%$, enquanto o APD médio foi de $9 \%$ (Tabela 3 ). Esse resultado corrobora os trabalhos comparando o PPD e APD no Brasil (LAMBERTS et al., 2013), demonstrando que o PPD não prediz adequadamente a insatisfação térmica real das pessoas (APD).

A Figura 12 apresenta a relação entre a porcentagem real de pessoas insatisfeitas (APD) e o voto médio de sensação térmica real das pessoas (AMV) para cada estudo de campo. Comparandose a relação do PMV com o PPD (Figura 11) podese perceber que com um PMV igual a -0,50 o PPD é aproximadamente $10 \%$. Entretanto, o valor mínimo de AMV médio foi de -0,48 (Figura 12), o que resultou em um APD de $22 \%$, bem acima do predito pelo modelo de Fanger (1970).

Figura 10 - Correlação entre o voto médio predito (PMV) e o voto médio real (AMV)

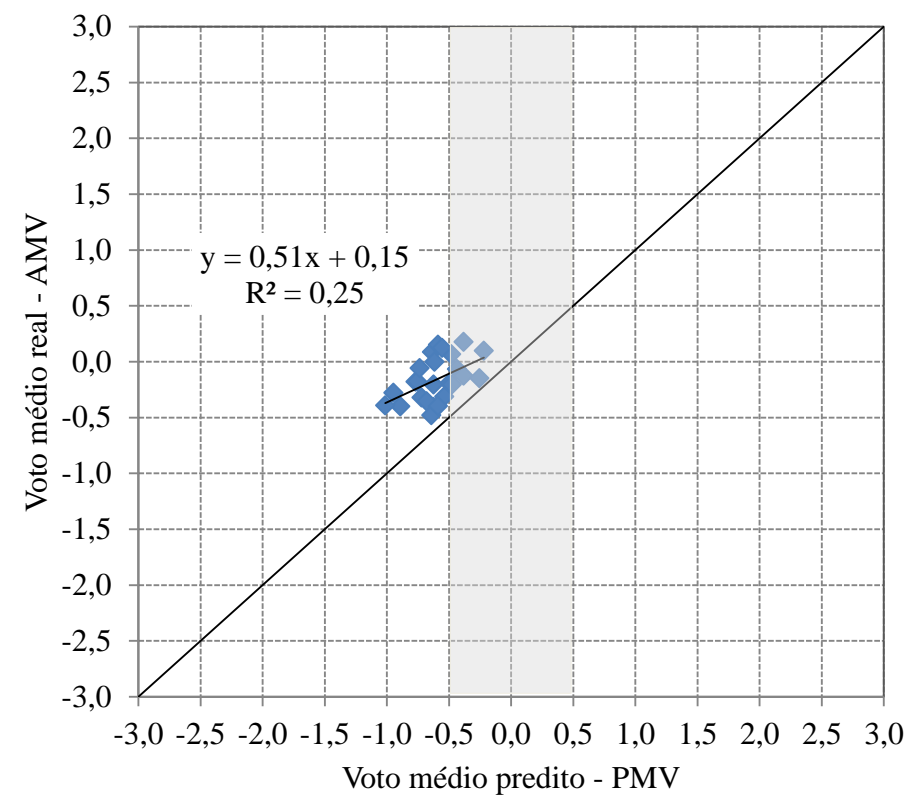

120 Rupp, R. F.; De Vecchi, R.; Asmus, B. F.; Candido, C. M.; Ghisi, E. 
Figura 11 - Porcentagem real de pessoas insatisfeitas (APD) e porcentagem predita de insatisfeitos (PPD) em função do PMV
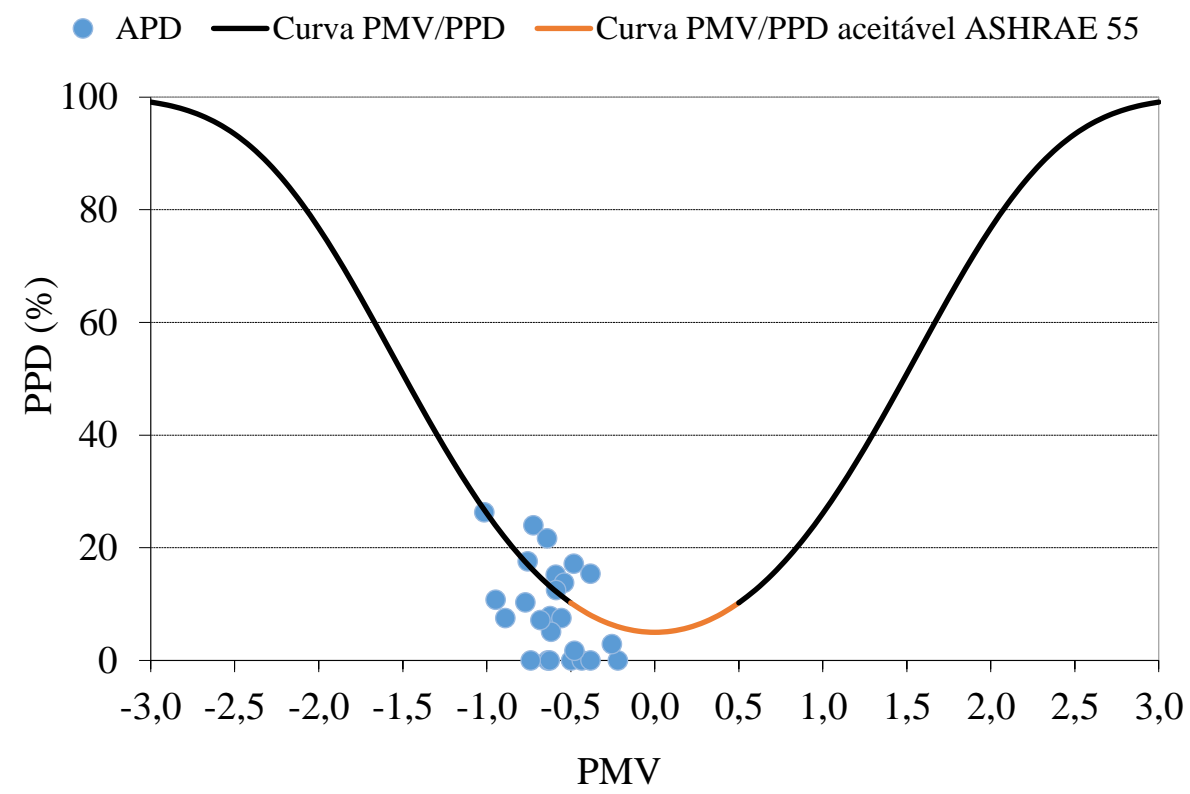

Figura 12 - Porcentagem real de pessoas insatisfeitas (APD) em função do voto médio de sensação térmica (AMV)

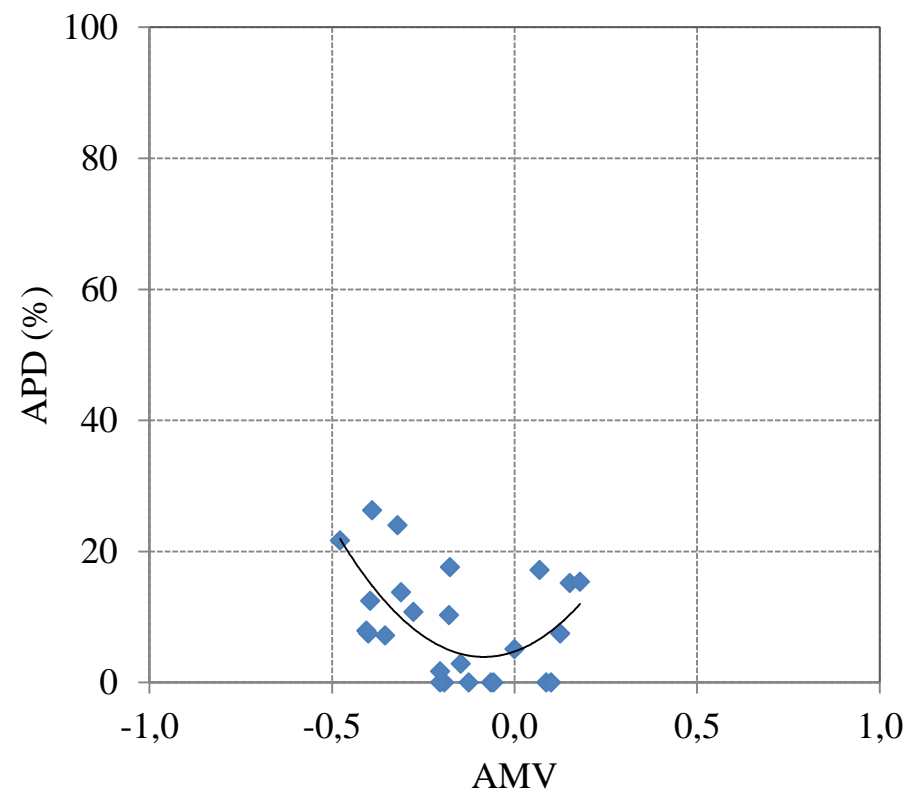

\section{Conclusões}

Este trabalho discutiu os resultados de campo de um estudo conduzido na cidade de Florianópolis, SC, que envolveu 284 usuários e 1.235 votos de sensação, preferência e aceitabilidade térmica e da velocidade do ar, em uma edificação de escritórios que opera predominantemente com sistema central de condicionamento de ar.

Com base nos resultados apresentados é possível concluir que: (a) sobre sensação e preferência térmica: a maioria das pessoas avaliou o ambiente térmico como neutro e gostaria de manter as condições térmicas presenciadas durante os estudos de campo;

(b) sobre aceitabilidade térmica e conforto térmico: o ambiente térmico interno da edificação estudada foi avaliado como aceitável e confortável pela grande maioria das pessoas $(95 \%$ e $90 \%$, respectivamente). Entretanto, o desconforto térmico por frio, principalmente nas mãos e nos 
pés, foi reportado por algumas pessoas, mesmo quando a velocidade do ar foi avaliada como aceitável, apesar de baixa durante toda a medição. Tais resultados enfatizam a importância da utilização de sistemas de condicionamento personalizados, comumente empregados para a otimização de espaços térmicos localizados, diminuindo as diferenças relacionas à subjetividade dos usuários (VESELÝ; ZEILER, 2014; ZHANG; ARENS; ZHAI, 2015);

(c) sobre adequabilidade do modelo analítico das normas de conforto internacionais: o método proveniente do PMV/PPD superestimou a sensação de frio dos usuários nas condições em que os estudos de campo foram realizados (outono, inverno e primavera, com velocidade do ar baixa e temperaturas controladas próximas de $23^{\circ} \mathrm{C}$ durante todo o ano). O modelo de Fanger também não estimou adequadamente a porcentagem de insatisfação térmica das pessoas. Os resultados deste estudo sugeriram a inadequação de tal modelo no clima em questão, principalmente quando se considera o método proveniente das normas internacionais que adotaram como confortável o intervalo de PMV entre $\pm 0,50$; e

(d) os altos níveis de conforto térmico e aceitabilidade térmica na edificação estudada, combinados com a leve sensação de frio observada durante todos os estudos de campo, apesar das baixas velocidades do ar presenciadas pelos usuários, sugerem que existe uma possibilidade de aumentar o setpoint de temperatura (atualmente entre 22 e $24^{\circ} \mathrm{C}$ ). Dessa forma, poderia haver economia de energia por meio do uso do sistema de ar condicionado.

Embora as conclusões deste trabalho se limitem à amostra e condições ambientais estudadas, elas poderiam ser extrapoladas para cidades de clima similar, considerando edifícios que operam com ar condicionado durante $o$ ano inteiro $e$ as temperaturas operativas internas mantidas em um intervalo similar (entre $22^{\circ} \mathrm{C}$ e $24^{\circ} \mathrm{C}$ ).

\section{Referências}

\section{AMERICAN SOCIETY OF HEATING, REFRIGERATING AND AIR-CONDITIONING ENGINEERS. Standard 55: thermal} environmental conditions for human occupancy. Atlanta, 2013.

ANDREASI, W. A.; LAMBERTS, R.; CÂNDIDO, C. Thermal Acceptability Assessment in Buildings Located in Hot Humid Regions in Brazil. Building and Environment, v. 45, n. 1, p. 1225-1232, 2010.
CÂNDIDO, C. et al. Air Movement Acceptability Limits and Thermal Comfort in Brazil's Hot Humid Climate Zone. Building and Environment, v. 45, n. 1, p. 222-229, 2010.

CAO, B. et al. Field Study of Human Thermal Comfort and Thermal Adaptability During the Summer and Winter in Beijing. Energy and Buildings, v. 43, n. 5, p. 1051-1056, 2011.

DAGHIGH, R.; SOPIAN, K. Effective Ventilation Parameters and Thermal Comfort Study of Airconditioned Offices. American Journal of Applied Sciences, v. 6, n. 5, p. 943-951, 2009.

DE DEAR, R. J.; BRAGER, G. Developing an Adaptive Model of Thermal Comfort and Preference. ASHRAE Transactions, v. 104, p. 145-167, 1998.

DEUBLE, M.; DE DEAR, R. Mixed-Mode Buildings: a double standard in occupants' comfort expectations. Building and Environment, v. 54, p. 53-60, 2012.

FANGER, P. O. Thermal Comfort: analysis and applications in environmental engineering. Copenhagen: Danish Technical Press, 1970.

FONG, K. F.; CHOW, T. T.; LI, C. Comfort Zone of Air Speeds and Temperatures for Airconditioned Environment in the Subtropical Hong Kong. Indoor and Built Environment, v. 19, n. 3, p. 375-381, 2010.

HENS, H. S. L. C. Thermal Comfort in Office Buildings: two case studies commented. Building and Environment, v. 44, n. 7, p. 1399-1408, 2009.

HOYT, T. et al. CBE Thermal Comfort Tool. Center for the Built Environment, University of California Berkeley, 2013. Disponível em: $<$ http://cbe.berkeley.edu/comforttool/>. Acesso em: 05 mar. 2014.

HUMPHREYS, M. A.; RIJAL, H. B.; NICOL, J. F. Updating the Adaptive Relation Between Climate and Comfort Indoors: new insights and an extended database. Building and Environment, v. 63, p. 40-55, 2013.

HUMPHREYS, M. A.; NICOL, J.F. The Validity of ISO-PMV for Predicting Comfort Votes in Every-Day Thermal Environments. Energy and Buildings, v. 34, p. 667-684, 2002.

HWANG, R.-L. et al. Additive Model for Thermal Comfort Generated by Matrix Experiment usiNg Orthogonal Array. Building and Environment, v. 44, n. 8, p. 1730-1739, 2009. 
INTERNATIONAL STANDARD

ORGANIZATION. ISO 7730: ergonomics of the thermal environment: analytical determination and interpretation of thermal comfort using calculation of the PMV and PPD indices and local thermal comfort criteria. Atalnta, 2005.

KUCHEN, E.; FISCH, M. N. Spot Monitoring: thermal comfort evaluation in 25 office buildings in winter. Building and Environment, v. 44, n. 4, p. 839-847, 2009.

LAMBERTS, R. et al. Towards a Brazilian Standard on Thermal Comfort. 2013. Disponível em:

<http://www.labeee.ufsc.br/sites/default/files/publi cacoes/relatorios_pesquisa/RP_Towards_a_Brazili an_Standard_Complete_Version.pdf $>$. Acesso em: 2 fev. 2016.

MAITI, R. Physiological and Subjective Thermal Response from Indians. Building and

Environment, v. 70, p. 306-317, 2013.

MAITI, R. PMV Model is Insufficient to Capture Subjective Thermal Response from Indians. International Journal of Industrial Ergonomics, v. 44, n. 3, p. 349-361, 2014.

NICOL, F.; HUMPHREYS, M. Derivation of the Adaptive Equations for Thermal Comfort in FreeRunning Buildings in European Standard EN15251. Building and Environment, v. 45, n. 1, p. 11-17, 2010.
NICOL, J. F.; HUMPHREYS, M. A. Adaptive Thermal Comfort and Sustainable Thermal Standards for Buildings. Energy and Buildings, v. 34, n. 6, p. 563-572, 2002.

RICCIARDI, P.; BURATTI, C. Thermal Comfort in Open Plan Offices in Northern Italy: an adaptive approach. Building and Environment, v. 56, p. 314-320, 2012.

RUPP, R. F.; VÁSQUEZ, N.G.; LAMBERTS, R. A Review of Human Thermal Comfort in the Built Environment. Energy and Buildings, v. 105, p. 178-205, 2015.

VAN HOOF, J. Forty Years of Fanger's Model of Thermal Comfort: comfort for all? Indoor Air, v. 18, p. 182-201, 2008.

VESELÝ, M.; ZEILER, W. Personalized Conditioning and Its Impact on Thermal Comfort and Energy Performance: a review. Renewable and Sustainable Energy Reviews, v. 34, p. 401408, 2014.

ZHANG, H.; ARENS, E.; ZHAI, Y. A Review of the Corrective Power of Personal Comfort Systems in Non-Neutral Ambient Environments. Building and Environment, v. 91, p. 15-41, 2015.

\section{Agradecimentos}

Os autores agradecem a CAPES e ao CNPq pelas bolsas de estudo que lhes permitiram realizar esta pesquisa.

Ricardo Forgiarini Rupp

Laboratório de Eficiência Energética em Edificações | Universidade Federal de Santa Catarina | Trindade | Caixa Postal 476 |

Florianópolis - SC - Brasil | CEP 88040-970 | Tel.: (48) 3721-5184 | E-mail: ricardorupp@gmail.com

Renata De Vecchi

Laboratório de Eficiência Energética em Edificações | Universidade Federal de Santa Catarina | Tel.: (48) 3271-5185 |

E-mail: renata.vecchi@labeee.ufsc.br

Bernardo Farias Asmus

Laboratório de Eficiência Energética em Edificações | Universidade Federal de Santa Catarina | Tel.: (48) 3271-5185 |

E-mail: bernardoasmus@gmail.com

Christhina Maria Cândido

Faculty of Architecture, Design and Planning | The University of Sydney | Wilkinson bd (G04), Room 587, Darlington | Sydney - Austrália | 2006 | Tel. +(2) 9351-3206 | E-mail: christhina.candido@sydney.edu.au

Enedir Ghisi

Laboratório de Eficiência Energética em Edificações | Universidade Federal de Santa Catarina | Tel.: (48) 3721-2115 |

E-mail: enedir@labeee.ufsc.br

Revista Ambiente Construído

Associação Nacional de Tecnologia do Ambiente Construído

Av. Osvaldo Aranha, $99-3^{\circ}$ andar, Centro

Porto Alegre - RS - Brasil

CEP 90035-190

Telefone: +55 (51) 3308-4084

Fax: +55 (51) 3308-4054

www.seer.ufrgs.br/ambienteconstruido

E-mail: ambienteconstruido@ufrgs.br 